\title{
Alcohol y cerebro adolescente
}

\section{Alcohol and adolescent brain}

\author{
Fernando CadaveIRA Mahía*
}

*Profesor Titular de Psicobiología. Departamento de Psicoloxía Clínica e Psicobioloxía, Universidade de Santiago de Compostela

Enviar correspondencia a:

Fernando Cadaveira Mahía. Departamento de Psicoloxía Clínica e Psicobioloxía. Rúa Xosé María Suárez Núñez, s/n. Campus Sur. 15782 Santiago de Compostela. Teléfono 981563100 ext 13797. Fax 981528071 . Correo electrónico: fernando.cadaveira@usc.es

\section{RESUMEN}

El patrón de consumo intensivo intermitente de alcohol (Binge Drinking) se ha consolidado entre adolescentes y jóvenes. Este patrón (BD) ha despertado una fuerte preocupación en la comunidad neurocientifica por su posible impacto en un cerebro aún en maduración. La investigación con modelos animales demuestra los graves efectos estructurales y funcionales que un patrón $\mathrm{BD}$ puede tener a estas edades. Algunas regiones como el cortex prefrontal y el hipocampo, de maduración más tardía, y los importantes procesos que éstas organizan, parecen diana especial de la acción del alcohol. Los escasos estudios realizados en humanos van en la dirección de lo adelantado por la investigación animal. Sin embargo, estamos lejos de poder responder si este patrón $\mathrm{BD}$ afecta de forma especial al cerebro adolescente y de precisar cuáles son sus consecuencias a corto y largo plazo. Son limitaciones habituales en los estudios el insuficiente control de poderosos factores de confusión, la utilización de diseños que no permiten diferenciar entre marcadores y efectos, el uso de muestras reducidas o la ausencia de réplicas. Aunque se hace insostenible una hipótesis de inocuidad y se acumula evidencia que nos advierte sobre los peligros de esta forma de consumo, se ha producido en general una cierta sobreinterpretación de los resultados. A la valiosa contribución realizada por estudios transversales debe seguir la de estudios longitudinales, en muestras de tamaño apropiado, con mayor control de variables críticas, que ayuden a identificar qué estructuras están más afectadas y cuáles son las consecuencias neurocognitivas a corto y largo plazo.

Palabras clave: Consumo intensivo de alcohol, adolescencia, cerebro, efectos neurocognitivos.

\section{ABSTRACT}

Binge Drinking (BD) pattern is firmly established among adolescents and young people. This pattern has raised a strong concern within the neuroscience community due to its possible impact on specific brain regions still maturing. Research with animal models demonstrates that BD may have serious structural and functional effects during these ages. Some regions like the prefrontal cortex or the hippocampus and the cognitive processes in which these structures play a role seem to be specific targets of alcohol. Results provided by the scarce studies carried out in humans support the evidence from previous animal experiments. However, we are far from being able to answer if this pattern of consumption has a special effect in the adolescent brain and what the short and long-term consequences are. Common limitations in human studies include the insufficient control of powerful confounding factors, the use of designs that does not allow differentiation between markers and effects, the use of reduced samples and the lack of replication studies. Although the innocuousness hypothesis is no longer sustained and experimental evidence has accumulated showing the risk associated with $B D$, results have been somewhat overinterpretated. The valuable contributions of cross-sectional designs should be complemented by the contributions of prospective longitudinal studies with appropriated sample sizes and a higher control of critical variables in order to identify short and long-term structural and neurocognitive consequences.

Key words: alcohol, binge drinking, adolescence, brain, cognitive function. 


\section{CONSUMO INTENSIVO DE ALCOHOL EN FIN DE SEMANA}

L a adolescencia supone un tránsito a la adultez en la que la propia maduración biológica afecta y se ve afectada por variables de diverso orden (cognitivas, afectivas, psicosociales,...). A nivel cerebral ésta abarca la segunda década de la vida, en la que se producen cambios neuromadurativos importantes, extendiéndose para algunas estructuras de evolución más reciente hasta aproximadamente los 25 años. En contra de lo que se presuponía hace un par de décadas, el cerebro adolescente parece especialmente vulnerable a los efectos del alcohol siendo probablemente las regiones de maduración más tardia diana especial. Este hecho pasa a ser muy preocupante a la luz de los datos de prevalencia que el consumo intensivo de fin de semana tiene entre adolescentes y jóvenes en Europa y, especialmente, en España. Informes recientes del Observatorio Español sobre Drogas han destacado la consolidación de un patrón de consumo intermitente, que se concentra en sesiones de pocas horas y en los días de fin de semana'. Este patrón se caracteriza por su alta prevalencia en jóvenes, igualación del consumo entre sexos, rejuvenecimiento del perfil de bebedores abusivos y escasa percepción del riesgo. Es lo que la literatura anglosajona ha dado en Ilamar "binge drinking" (BD) y que como criterio más consensuado ${ }^{2}$ supone beber 5 o más UBEs (Unidad de Bebida Estándar) para hombres y 40 más para mujeres en una misma sesión.

Según el último informe de la encuesta estatal sobre uso de drogas en estudiantes de enseñanzas secundarias' ${ }^{1}$, el alcohol es la sustancia cuyo consumo está más extendido entre los 14 y 18 años. Aproximadamente, un 53,4\% de los estudiantes de estas edades ( $47,3 \%$ chicas y $59,8 \%$ chicos) han tomado en los últimos 30 días 5 o más cañas/copas de bebidas alcohólicas en la misma ocasión (BD). Un 17,7\% lo ha hecho más de 4 veces el último mes, siendo habitual que alcancen la embriaguez. Estos datos son medias para ese grupo de edad que se ven muy incrementados entre los 18 y los 24 años, especialmente en población universitaria, como se ha podido comprobar, entre otros, en nuestro estudio.

\section{OPORTUNIDAD PARA OTROS FACTORES DE RIESGO: SEXO, INICIO TEMPRANO, POLICONSUMO}

Además de los efectos directos que un patrón $\mathrm{BD}$ pueda tener sobre la estructura y la función cerebral, la práctica de esta forma de consumo se erige en oportunidad para la acción de otros factores de riesgo.

\section{Diferencias sexuales}

Aunque con algunos informes discrepantes ${ }^{3,4}$, la mayor parte de las investigaciones sugieren que las mujeres son más vulnerables a los efectos del alcohol, argumentando, entre otras, razones de orden metabólico y hormonal. En adolescentes y jóvenes, en un estudio reciente con una muestra de 14 a 17 años con problemas por abuso de alcohol, se informó de patrones de actividad cerebral más anómalos mediante RMNf (resonancia magnética nuclear funcional) en mujeres que estaban realizando pruebas de memoria de trabajo espacial ${ }^{5}$. Empleando pruebas neuropsicológicas otras investigaciones llegaron a conclusiones semejantes en jóvenes $\mathrm{BD}^{6}$. Además de los factores antes mencionados, se han esgrimido diferencias sexuales en procesos neuromadurativos. Dada la importante incorporación de las adolescentes y jóvenes a este patrón de consumo de fin de semana ${ }^{7}$, es imprescindible valorar si esta equiparación paulatina implica complicaciones asociadas al sexo.

\section{Edad de inicio}

El inicio temprano se ha asociado a un incremento importante del riesgo a desarrollar alcoholismo. Así, se ha informado de hasta cuatro veces más probabilidad para desarrollar dependencia en jóvenes con inicio al consumo antes de los 15 años respecto a jóvenes con inicio a partir de los $20^{8}$. Aún así, los resultados no son siempre coherentes, mayor vulnerabilidad pero también mayor capacidad para recuperarse podrian coexistir. Esta importante cuestión sólo se puede abordar mediante estudios longitudinales. En nuestro país, existen razones para la preocupación; la última encuesta escolar indica una media de inicio de 13,9 años (muchos se acercan a los 12); éste es un fuerte motivo para investigar las posibles consecuencias. Desafortunadamente, para muchos adolescentes el consumo de alcohol supone una forma de socializarse que no se acompaña de una especial percepción de riesgo.

\section{Policonsumo}

Para muchos especialistas, el alcohol es una sustancia de entrada al consumo de otras drogas. Sin entrar en la cuestión de causalidad o concomitancia respecto a factores previos de riesgo, los datos de prevalencia de policonsumo entre jóvenes BD son muy consistentes. En nuestra propia investigación con estudiantes universitarios de 18 a 19 años ${ }^{9}$ se comprueba alta prevalencia de policonsumo de cannabis (alcanza el $50 \%$ ) y otras sustancias de abuso (entre el 1 y el $5 \%$, dependiendo que sustancia).

\section{DE LA INQUIETUD SOCIAL A LA ALARMA CIENTIIFICA}

\section{Un patrón con elevados costes sociales}

La generalización de un patrón de consumo intermitente, intensivo, ligado al ocio y la cada vez más temprana edad de inicio fueron factores que empezaron a despertar inquietud 
en nuestra sociedad, especialmente por su asociación con el fenómeno del botellón. Los problemas ocasionados por este tipo de patrón suponen importantes pérdidas a nivel personal, familiar y social y han llegado a constituir una de las prioridades de actuación de los países desarrollados, entre ellos los de la Unión Europea (European Alcohol Action Plan 2000-2005). En este último ámbito se ha llegado a estimar el coste económico del abuso de alcohol entre el $2 \%$ y el $5 \%$ del PIB, según que países ${ }^{10}$.

Una cierta inquietud social por las consecuencias de esta forma de consumo, especialmente en Estados Unidos y otros países de la Unión Europea, antecedió a la propia alarma científica acerca de los efectos en el cerebro adolescente. De hecho, durante un buen número de años algunos índices comportamentales (efectos sedativos, sobre coordinación motora) llevaron a pensar que los jóvenes eran menos vulnerables a la acción del alcohol. Las principales variantes de la hipótesis del envejecimiento prematuro partian de este supuesto y apuntaban a más allá de los 40 años como etapa especialmente crítica"11.

La constatación de un inicio cada vez más temprano en el consumo y los estudios sobre vulnerabilidad genética para el alcoholismo tal vez fueron los factores que más poderosamente reorientaron la atención de la comunidad científica hacia el consumo en adolescentes y jóvenes. De entre todas las variables habitualmente consideradas (extensión de la historia de abuso, niveles de consumo,...) era únicamente la edad de inicio la variable que de forma consistente se asociaba a la gravedad de las complicaciones por consumo abusivo de alcohol. La incidencia de factores genéticos en la irrupción de formas de alcoholismo temprano de evolución rápida era otro dato que incrementaba la preocupación por la cada vez mayor precocidad en el consumo.

\section{Algunos estudios con modelos animales dispararon todas las alarmas}

Uno de los aspectos más relevantes y preocupantes de la alta prevalencia de consumo intensivo de alcohol en jóvenes es el efecto que esta forma de consumo muy probablemente tenga en la estructura y la función del un cerebro aún en desarrollo. Consecuencias que bien pudieran persistir a largo plazo.

A pesar de la precoz propuesta realizada por Hunt para humanos ${ }^{12}$, sería la investigación con modelos animales la que aportó las primeras evidencias firmes. En estos estudios se ha puesto el énfasis esencialmente en tres características diferenciales del patrón $\mathrm{BD}$ : inicio temprano, picos de consumo muy elevados en breve tiempo e intermitencia (múltiples retiradas). El efecto diferencial de estos tres factores respecto a formas de consumo más regulares en adultos fue estudiado en un buen número de laboratorios. Asi, a nivel estructural se ha podido observar que los cerebros de ratas adolescentes con un patrón BD muestran daños considerables en el córtex prefrontal, el córtex piriforme anterior y la corteza perirrinal, áreas que se corresponden con regiones de la corteza orbitofrontal y temporal, mientras que en ratas adultas también BD no se observa degeneración en esas regiones ${ }^{13}$. Otra estructura especialmente vulnerable es el hipocampo; así, en ratas adolescentes se observó bloqueo de los receptores NMDA-glutamato y del fenómeno de potenciación a largo plazo ${ }^{14,15}$; otros estudios han demostrado inhibición de la neurogénesis ${ }^{16}$. En España se informó de consecuencias comportamentales a largo plazo por daño en hipocampo, neocórtex y cerebelo derivado de procesos inflamatorios en ratas adolescentes con patrón de consumo BD ${ }^{17}$. Además del efecto que el alcohol en altas concentraciones o sus metabolitos puedan tener, el propio efecto de sufrir múltiples retiradas puede jugar un papel importante. Como demuestran algunos estudios, no es necesario esperar varios años para que se produzcan importantes efectos de un consumo intensivo e intermitente en ratas, bastan pocas sesiones para observar daño estructural en algunas cortezas olfativas e hipocampo ${ }^{13,18,19}$. Si bien las dosis necesarias para provocar en tan pocas sesiones estos daños no son equiparables a las del consumo humano, algunos de los efectos recuerdan los observados en alcohólicos crónicos.

A nivel neurocognitivo, se ha podido comprobar que las alteraciones producidas en procesos de aprendizaje y en la memoria espacial son más potentes en ratas adolescentes ${ }^{20}$. También se han informado de posibles consecuencias a largo plazo en ratas adultas que durante la adolescencia fueron sometidas a un patrón BD. Estas consecuencias eran especialmente evidentes en pruebas que implicaban a la memoria de trabajo ${ }^{21}$, aunque también fueron observadas dificultades persistentes en pruebas de aprendizaje y memoria espacial ${ }^{22}$. Una buena revisión de estos trabajos se encuentra en White y Swartzwelder ${ }^{23}$.

\section{Estudios en humanos}

En términos de maduración cerebral se considera que la adolescencia abarca la segunda década de la vida y para algunas estructuras buena parte de la tercera. De forma similar a lo que ocurre en otros períodos evolutivos críticos, en algunas regiones del cerebro adolescente se producen importantes transformaciones tanto regresivas (con pérdida de un número sustancial de sinapsis superfluas), como progresivas (de mielinización de las neuronas y consolidación de sus conexiones) que tendrán importantes consecuencias comportamentales en la vida adulta. Estas regiones en maduración median importantes aspectos del comportamiento adolescente y parecen diana especial para los efectos del alcohol ${ }^{24}$. Entre ellas debemos destacar la corteza prefrontal ${ }^{25}$. Los procesos neuromadurativos en esta región se extienden hasta la tercera década de la vida y tiene importantes repercusiones en funciones cognitivas relevantes como planificación, toma de decisiones, control comportamental, memoria de trabajo o procesos de atención, entre otros ${ }^{26}$. El abuso de alcohol en la adolescencia también ha sido asociado a menor volumen en cortex prefrontal, sin embargo esas diferencias podrian estar moduladas por el sexo, relacionadas con factores 
premórbidos o condicionadas genéticamente ${ }^{27}$. En cuanto a otra de las posibles dianas, el hipocampo, se trata de una estructura con enorme plasticidad que está implicada en la formación de nuevas memorias y en el mantenimiento de representaciones del entorno espacial. Como se ha señalado, algunos fenómenos asociados especialmente al hipocampo, como neurogénesis o potenciación a largo plazo, demostraron ser muy vulnerables a los efectos de un patrón BD en estudios en animales adolescentes o jóvenes ${ }^{15,28}$; el propio hipocampo muestra daño estructural después de escasas sesiones de ingesta de alcohol ${ }^{13}$. A nivel estructural, los escasos estudios en humanos ratifican algunos de los hallazgos observados en animales. Así, se ha informado, mediante RMN (resonancia magnética nuclear), de una reducción media significativa en el volumen del hipocampo en jóvenes que empezaban a cumplir los criterios DSMIV de abuso o dependencia alcohólica ${ }^{29}$. Otros trabajos destacan especialmente un menor volumen en hipocampo izquierdo $0^{30,31}$.

Los primeros estudios neuropsicológicos empiezan a aclarar la asociación de un patrón BD en adolescentes y jóvenes con bajo rendimiento en varios ámbitos. Algunos de estos trabajos fueron realizados por S. Tapert y S. Brown en San Diego (se pueden consultar las siguientes revisiones de las propias autoras ${ }^{32-34}$ ). Adolescentes que acumulaban al menos 100 sesiones BD obtuvieron peores rendimientos en test de aprendizaje, memoria y funcionamiento visuoespacial ${ }^{35}$. En un estudio de seguimiento efectuado durante 8 años (desde los 16 a los 24) se comprobó un efecto acumulativo que se trasladaba a los resultados en pruebas de aprendizaje y memoria. Desafortunadamente, añadido al problema de consumo de alcohol en muchos casos se presentaban otros policonsumos o estaba ya establecida dependencia al alcohol, por lo que no es posible discernir los efectos asociados a cada uno de estos factores ${ }^{36}$. Aunque la mayor parte de los estudios mediante potenciales evocados se centraron en pacientes alcohólicos y en la búsqueda de marcadores de riesgo, recientemente también se ha informado de anomalias en algunos componentes de potenciales evocados en jóvenes de 18-25 años con un patrón $\mathrm{BD}$, estas anomalias fueron interpretadas en términos de déficits inhibitorios centrales ${ }^{37}$; desafortunadamente, el diseño de esta investigación no permite descartar la posible influencia de variables como la historia de consumo familiar. En nuestro país algunos estudios con universitarios empiezan a ofrecer resultados interesantes; así, Garcia Moreno y colaboradores han informado recientemente en esta misma revista ${ }^{38}$ de bajo rendimiento en algunas tareas neuropsicológicas asociadas a procesos mediados por la corteza prefrontal. Nuestra investigación con una muestra de similares características, en las que se intenta controlar importantes fuentes de confusión, ofrece algunos resultados convergentes en pruebas neuropsicológicas, también encuentra diferencias significativas en componentes de potenciales evocados e hipoactividad en corteza prefrontal (BA 10) en tareas que demandan memoria de trabajo ${ }^{39,40}$.

\section{Posibles consecuencias a largo plazo}

Aunque la prevalencia de episodios BD suele alcanzar su plateau en torno a los 24-25 años, este patrón de consumo, conjuntamente con otras variables, constituye en sí una trayectoria de alto riesgo para acabar desarrollando problemas asociados al abuso de alcohol y/o al alcoholismo ${ }^{41}$. Si bien queda aún mucha investigación que realizar sobre las consecuencias de este patrón en humanos, el consumo intensivo en adolescentes puede conllevar consecuencias a largo plazo a nivel neurocognitivo. En modelos animales, se ha informado que la exposición a repetidas sesiones $\mathrm{BD}$ ocasiona consecuencias a largo plazo especialmente en tareas de memoria ${ }^{21}$. Los muy escasos estudios realizados con humanos una vez más parecen ir en la misma dirección ${ }^{42}$. La mayor propensión de los adolescentes y jóvenes hacia patrones de consumo $\mathrm{BD}$ y la correspondiente reiteración de pequeñas retiradas, podría tener importantes efectos en estas funciones. Estas consecuencias estarán muy probablemente moduladas a nivel individual por otros factores como riesgo genético, sexo, edad de inicio o policonsumo.

\section{LA NECESIDAD DE REDUCIR POSIBLES FUENTES DE CONFUSIÓN EN LA INVESTIGACIÓN FUTURA}

El consumo abusivo de alcohol y el desarrollo de problemas asociados a él es el resultado de la interacción entre factores ambientales y genéticos, en algún caso relacionados con problemas comportamentales de más amplio espectro que favorecen conductas de riesgo. Causas y consecuencias, marcadores e índices de los efectos de la sustancia son difíciles de discernir en nuestra especie dadas las limitaciones propias de la investigación en humanos. Así, por ejemplo, distintos grupos hemos podido constatar en hijos de alcohólicos, previamente a cualquier contacto con la sustancia, la presencia de índices neurofisiológicos y neuropsicológicos que previamente habían sido interpretados como indicadores del efecto del consumo crónico de alcohol en adultos. Algunos de estos indicadores son interpretados ahora como manifestación de retrasos madurativos, perfiles de personalidad, trastornos de conducta o psicopatología, todos ellos factores que han sido asociados con el abuso de alcohol y otras sustancias psicoactivas. A modo de ejemplo, diferencias en la función frontal no sólo se asocian al consumo intensivo de alcohol sino que también aparecen en poblaciones con alto riesgo, incluso de forma previa a haberse iniciado cualquier consumo ${ }^{43,44}$. Si bien existen evidencias procedentes de estudios en animales y se acumula datos acerca de una asociación entre consumo intensivo de alcohol y algunos indices de funcionamiento neurocognitivo en humanos, la investigación realizada hasta el momento aún no aporta datos definitivos sobre cuáles son las consecuencias de este patrón en el cerebro adolescente.

Por otra parte, la caracterización del desarrollo a nivel neuroestructural y neurofuncional en nuestra especie es aún insuficiente para poder establecer una buena base de 
comparación. A un contexto de investigación esencialmente clínico probablemente deba añadirse otro asentado en un marco conceptual del desarrollo ${ }^{45-47}$. Dificultades derivadas de esa insuficiente caracterización, limitaciones propias de los diseños, muestras reducidas y falta de control sobre algunas variables críticas nos pueden conducir fácilmente a generalizaciones imprudentes, a confundir marcadores con efectos o a mezclar consecuencias del consumo alcohol con los efectos de otros factores. Entre estas variables críticas fuentes de confusión conviene destacar especialmente la falta de control de vulnerabilidad genética, presencia de trastornos psicológicos y los efectos del consumo de otras sustancias psicoactivas. En cuanto a la vulnerabilidad genética, un buen numero de estudios, entre los que se encuentra nuestra propia investigación, han encontrado de forma consistente diferencias neuropsicológicas y psicofisiológicas entre niños procedentes de familias con alta densidad de alcoholismo y sus controles de bajo riesgo, especialmente en tareas que implican memoria de trabajo y capacidades visuoespaciales ${ }^{43,48-50}$, procesos que se ha informado están afectados por el patrón BD. También es común en los estudios sobre patrón BD ausencia de control en las muestras de trastornos psicológicos, especialmente depresión o trastornos de alimentación en mujeres y trastornos de conducta en hombres ${ }^{51}$. Es muy frecuente, por último, detectar en las muestras presencia de policonsumo y sin embargo atribuir las diferencia al consumo de alcohol. En el estudio epidemiológico que hemos desarrollado con población universitaria se puso de manifiesto un mayor consumo de medicamentos en los jóvenes $\mathrm{BD}$, también quedo clara una alta prevalencia de consumo de cannabis (55\% en hombres, $45 \%$ en mujeres) y, aunque a mucha distancia el policonsumo de otras sustancias, confirmando informes previos ${ }^{52}$. Sobre estas variables es necesario ejercer control.

A modo de conclusión, además de avanzar en algunas de las líneas indicadas para poder distinguir entre marcadores y efectos, o para excluir el papel jugado por otras variables como policonsumo, etc., debemos esperar aportaciones importantes de investigaciones en humanos que combinen técnicas estructurales y funcionales no invasivas o mínimamente invasivas con diseños longitudinales. La dificultad de esta empresa científica no debe leerse en clave de desanimo ni oscurecer una conclusión más que suficientemente asentada, aunque queda mucho por avanzar acerca de los efectos del alcohol sobre la estructura y la función cerebral en adolescentes una hipótesis de inocuidad o mayor resistencia hoy es insostenible. Tomada en su conjunto, existe fuerte evidencia desde la investigación en modelos animales y apoyo estimable desde investigación en humanos para afirmar que la práctica de un consumo intensivo de alcohol en fin de semana entraña un fuerte peligro para la salud de los jóvenes y les incorporan a una trayectoria de muy alto riesgo para desarrollar alcoholismo, incluso en ausencia de vulnerabilidad genética y factores premórbidos, como empezamos a vislumbrar en algunas investigaciones en las que nos esforzamos en controlar estas fuentes habituales de confusión.

\section{REFERENCIAS}

1. Observatorio Español sobre Drogas. Encuesta Estatal sobre uso de drogas en estudiantes de enseñanzas secundarias (ESTUDES) 2006-2007. Madrid. Ministerio de Sanidad y Consumo. Delegación del Gobierno para el Plan Nacional sobre Drogas, 2007.

2. National Institute on Alcohol Abuse and Alcoholism. NIAAA Council Approves Definitión of Binge Drinking. NIAAA Newsletter 2004; 3: 3 .

3. Pfefferbaum A, Rosenbloom M, Deshmukh A, Sullivan E. Sex differences in the effects of alcohol on brain structure. Am J Psychiatry 2001; 158: 188-97.

4. Wuethrich B. Neurobiology. Does alcohol damage female brains more? Science $(2001 ;$ 291: 2077-9.

5. Caldwell LC, Schweinsburg AD, Nagel BJ, Barlett VC, Brown SA, Tapert SF. Gender and adolescent alcohol use disorders on BOLD (blood oxygen level dependent) response to spatial working memory. Alcohol Alcohol 2000; 40: 194-200.

6. Townshend JM, Duka T. Binge drinking, cognitive performance and mood in a population of young social drinkers. Alcohol Clin Exp Res 2005; 29: 317-25.

7. Observatorio Español sobre Drogas. Informe de la Encuesta Domiciliaria sobre Alcohol y Drogas en España (EDADES) 20072008. Recuperado el 6 de febrero de 2009, de http://www.pnsd. msc.es/Categoria2/observa/pdf/InformeEdades2007-2008.pdf.

8. Grant BF, Dawson DA. Age at onset of alcohol use and its association with DSM-IV alcohol abuse and dependence: results from the National Longitudinal Alcohol Epidemiologic Survey. J Subst Abuse 1997; 9: 103-10.

9. Caamaño-Isorna $F$, Corral M, Parada M, Cadaveira F. Factors associated with risky alcohol use and heavy episodic drinking among Spanish university students. J Stud Alcohol Drugs 2008; 69: 308-12.

10. Andersson BH, B.; Beck, F.; Choquet, M.; Kokkevi, A.; Fotiou, A. Alcohol and Drug Use Among European 17-18 Year Old Students. Data from the ESPAD Project. Swedish Council for Information on Alcohol and Other Drugs (CAN), The Pompidou Group at the Council of Europe ed. Stockholm: Modintryckoffset AB 2007.

11. Cadaveira F, Roso M, Grau C, Sanchez-Turet M. Effects of age on event-related potentials in chronic alcoholics: a multimodal study. Neuropsychobiology. 1992; 25: 166-71.

12. Hunt WA. Are binge drinkers more at risk of developing brain damage? Alcohol 1993; 10: 559-61.

13. Crews FT, Braun CJ, Hoplight B, Switzer RC, Knapp DJ. Binge ethanol consumption causes differential brain damage in young adolescent rats compared with adult rats. Alcohol Clin Exp Res 2000; 24: 1712-23.

14. Swartzwelder HS, Wilson WA, Tayyeb MI. Differential sensitivity of NMDA receptor-mediated synaptic potentials to ethanol in immature versus mature hippocampus. Alcohol Clin Exp Res 1995; 19: 320-3.

15. Swartzwelder HS, Wilson WA, Tayyeb MI. Age-dependent inhibition of long-term potentiation by ethanol in immature versus mature hippocampus. Alcohol Clin Exp Res 1995; 19: 1480-5.

16. Crews FT, Mdzinarishvili A, Kim D, He J, Nixon K. Neurogenesis in adolescent brain is potently inhibited by ethanol. Neuroscience 2006; 137: 437-45.

17. Pascual M, Blanco AM, Cauli O, Minarro J, Guerri C. Intermittent ethanol exposure induces inflammatory brain damage and 
causes long-term behavioural alterations in adolescent rats. Eur J Neurosci 2007; 25: 541-50.

18. Collins MA, Corso TD, Neafsey EJ. Neuronal degeneration in rat cerebrocortical and olfactory regions during subchronic "binge" intoxication with ethanol: possible explanation for olfactory deficits in alcoholics. Alcohol Clin Exp Res 1996; 20: 284-92.

19. Crews F, ed. Neurotoxicity of alcohol: excitotoxicity, oxidative stress, neurotrophic factors, apoptosis, and cell adhesion molecules. Bethesda, MD.: NIAAA 2000.

20. Markwiese BJ, Acheson SK, Levin ED, Wilson WA, Swartzwelder HS. Differential effects of ethanol on memory in adolescent and adult rats. Alcohol Clin Exp Res 1998; 22: 416-21.

21. White AM, Ghia AJ, Levin ED, Swartzwelder HS. Binge pattern ethanol exposure in adolescent and adult rats: differential impact on subsequent responsiveness to ethanol. Alcohol Clin Exp Res 2000; 24: 1251-6.

22. Silvers JM, Tokunaga S, Mittleman G, Matthews DB. Chronic intermittent injections of high-dose ethanol during adolescence produce metabolic, hypnotic, and cognitive tolerance in rats. Alcohol Clin Exp Res 2003; 27: 1606-12.

23. White AM, Swartzwelder HS. Age-related effects of alcohol on memory and memory-related brain function in adolescents and adults. Recent Dev Alcohol 2005; 17: 161-76.

24. Crews F, He J, Hodge C. Adolescent cortical development: a critical period of vulnerability for addiction. Pharmacol Biochem Behav 2007; 86: 189-99.

25. Luna B, Sweeney JA. The emergence of collaborative brain function: FMRI studies of the development of response inhibition. Ann N Y Acad Sci 2004; 1021: 296-309.

26. Stuss DT, Levine B. Adult clinical neuropsychology: lessons from studies of the frontal lobes. Annu Rev Psychol 2002; 53: 40133.

27. Medina KL, McQueeny T, Nagel BJ, Hanson KL, Schweinsburg AD, Tapert SF. Prefrontal cortex volumes in adolescents with alcohol use disorders: unique gender effects. Alcohol Clin Exp Res 2008; 32: 386-94.

28. Pyapali GK, Turner DA, Wilson WA, Swartzwelder HS. Age and dosedependent effects of ethanol on the induction of hippocampal long-term potentiation. Alcohol 1999; 19: 107-11.

29. De Bellis MD, Clark DB, Beers SR, Soloff PH, Boring AM, Hall J, et al. Hippocampal volume in adolescent-onset alcohol use disorders. Am J Psychiatry 2000; 157: 737-44.

30. Medina KL, Schweinsburg AD, Cohen-Zion M, Nagel BJ, Tapert SF. Effects of alcohol and combined marijuana and alcohol use during adolescence on hippocampal volume and asymmetry. Neurotoxicol Teratol 2007; 29: 141-52.

31. Nagel BJ, Schweinsburg AD, Phan V, Tapert SF. Reduced hippocampal volume among adolescents with alcohol use disorders without psychiatric comorbidity. Psychiatry Res 2005; 139: 181-90.

32. Brown SA, Tapert SF. Adolescence and the trajectory of alcohol use: basic to clinical studies. Ann N Y Acad Sci 2004; 1021: 23444.

33. Tapert SF, Caldwell L, Burke C. Alcohol and the adolescent brain Human studies. Alcohol Res Health 2004; 28: 205-12.

34. Tapert SF, Schweinsburg AD. The human adolescent brain and alcohol use disorders. Recent Dev Alcohol 2005; 17: 177-97.

35. Brown SA, Tapert SF, Granholm E, Delis DC. Neurocognitive functioning of adolescents: effects of protracted alcohol use. Alcohol Clin Exp Res 2000; 24: 164-71.
36. Tapert SF, Granholm E, Leedy NG, Brown SA. Substance use and withdrawal: neuropsychological functioning over 8 years in youth. J Int Neuropsychol Soc 2002; 8: 873-83.

37. Ehlers CL, Phillips E, Finnerman G, Gilder D, Lau P, Criado J. P3 components and adolescent binge drinking in Southwest California Indians. Neurotoxicol Teratol 2007; 29: 153-63.

38. García-Moreno LM. Actividad prefrontal y alcoholismo de fin de semana en jóvenes. Adicciones 2008; 20: 271-80.

39. Crego A, Rodríguez Holguín S, Parada M, Mota-Miranda N, Corral M, Caamaño-Isorna $F$, et al. Actividad electrofisiológica relacionada con memoria de trabajo visual en jóvenes consumidores intensivos de alcohol Comunicación presentada en las XXXV Jornadas Nacionales de Socidrogalcohol. A Coruña, 2008.

40. Parada M, Mota-Miranda N, Corral M, Crego A, Fernández-Eiré $C$, Rodríguez Holguin $S$, et al. Consumo intensivo de alcohol y memoria de trabajo en jóvenes universitarios: resultados preliminares. Póster presentado en las XXXV Jornadas Nacionales de Socidrogalcohol. A Coruña, 2008.

41. Chassin L, Pitts SC, Prost J. Binge drinking trajectories from adolescence to emerging adulthood in a high-risk sample: predictors and substance abuse outcomes. J Consult Clin Psychol 2002; 70: 67-78.

42. Weissenborn R, Duka T. Acute alcohol effects on cognitive function in social drinkers: their relationship to drinking habits. Psychopharmacology 2003; 165: 306-12.

43. Corral MM, Holguin SR, Cadaveira F. Neuropsychological characteristics in children of alcoholics: familial density. J Stud Alcohol 1999; 60: 509-13.

44. Rodriguez Holguin S, Corral M, Cadaveira F. Event-related potentials elicited by infrequent non-target stimuli in young children of alcoholics: family history and gender differences. Alcohol Alcohol 1998; 33: 281-90.

45. Brown SA, McGue M, Maggs J, Schulenberg J, Hingson R, Swartzwelder $S$, et al. A developmental perspective on alcohol and youths 16 to 20 years of age. Pediatrics 2008; $121 \mathrm{Supl} 4$ : S290-310.

46. Clark DB, Tapert SF. Introduction to alcohol and adolescent brain development. Alcohol Clin Exp Res 2008; 32: 373-4.

47. Masten AS, Faden VB, Zucker RA, Spear LP. Underage drinking: a developmental framework. Pediatrics 2008; 121 Supl 4: S23551.

48. Berman SM, Noble EP. Reduced visuospatial performance in children with the D2 dopamine receptor A1 allele. Behav Genet 1995; 25: 45-58.

49. Corral M, Holguin SR, Cadaveira F. Neuropsychological characteristics of young children from high-density alcoholism families: a three-year follow-up. J Stud Alcohol 2003; 64: 195-9.

50. Rodriguez Holguin S, Corral M, Cadaveira F. Event-related potentials elicited by a visual continuous performance task in children of alcoholics. Alcohol 1999; 19: 23-30.

51. Sartor CE, Lynskey MT, Heath AC, Jacob T, True W. The role of childhood risk factors in initiation of alcohol use and progression to alcohol dependence. Addiction 2007; 102: 216-25.

52. Aarons GA, Brown SA, Coe MT, Myers MG, Garland AF, EzzetLofstram $R_{1}$ et al. Adolescent alcohol and drug abuse and health. J Adolesc Health 1999; 24: 412-21. 